\title{
Resistencias y costos unitarios de concretos fabricados con cementos utilizados en Huaraz, con agregados de la cantera Tacllán, y topex-concreto listo, Huaraz
}

Resistances and unit costs of concretes manufactured with cements used in Huaraz, with aggregates quarry Tacllán, and topex-concrete ready, Huaraz

\author{
Víctor Villegas Zamora ${ }^{1}$ y Miguel Corrales Picardo ${ }^{1}$
}

\section{RESUMEN}

La presente investigación ha tenido por objeto determinar y seleccionar la marca de cemento: Sol, Quisqueya, Inka empleando agregados de la cantera Tacllán, para la fabricación de concreto y topex-concreto listo; con lo cual busca lograr mayor resistencia a la compresión y optimizar los costos unitarios de fabricación en Huaraz.

Para el efecto, obtenidos los agregados de la cantera, se determinaron sus características físicas y empleando el método del Instituto Americano del Concreto, se ha realizado el diseño de mezclas de concreto con relación agua-cemento, para asentamiento entre 3" a 4"; para resistencias a compresión de 21 y $28 \mathrm{Mpa}$ se elaboraron y ensayaron briquetas de concreto en la máquina a compresión uniaxial, calculándose las resistencias. Los costos unitarios se determinaron en base a la cantidad de materiales requeridos por $\mathrm{yd}^{3}$ de concreto y precios de mercado. Se determinó que, para concretos diseñados para 21 y $28 \mathrm{Mpa}$, con cemento Quisqueya se ha logrado resistencias de 41.48 y $42.42 \mathrm{Mpa}$ y costos unitarios de 190.19 y 210.58 nuevos soles, respectivamente. Se reporta que, empleando cemento marca Quisqueya en la fabricación de concretos, se logra mayores resistencias y menores costos unitarios para resistencias de diseño 21 y $28 \mathrm{Mpa}$.

Palabras clave: concreto; agregados; cemento; resistencia; compresión; briquetas; costos.

\section{ABSTRACT}

The present research have to object determine the brand of cement: sun, quisqueya, Inca and using quarry aggregates Tacllán for the manufacture of concrete and concrete topex-ready; it is reached greater strength compressive and costs units manufacturing in Huaraz.

Obtained aggregates quarry, were determined their characteristics physical, using the method of the American Concrete Institute, was the design mix concrete with relation water-cement ratio, for settlement between 3" to 4"; for strengths compressive of 21 and $28 \mathrm{Mpa}$. Are developed and tested briquettes of concrete in machine uniaxial compression, calculating resistances. The costs units were determined based on the amount of materials required by $\mathrm{yd}^{3}$ of concrete and market prices. It was determined that, for concretes designed for 21 and $28 \mathrm{Mpa}$, with cement quisqueya managed resistances

1 Universidad Nacional Santiago Antúnez de Mayolo. Huaraz, Perú. 
of 41.48 and $42.42 \mathrm{Mpa}$, and costs units of 190.19 and 210.58 new suns, respectively. It is reported that using cement mark quisqueya in manufacturing concrete, they are achieved higher strength and lower unit costs; to resistances of design 21 and $28 \mathrm{Mpa}$.

Keywords: concrete; aggregates; cement; resistances; compression; briquettes; costs.

\section{ICHIKLLACHAW}

Waras markachaw, kunstruksiyun urya ruraykunachaw kunkritu rurashqakunam yaykun; Takllan kantirapa yapayninkunam, hina yaykun llutaypa kay laaya simintukuna: Inti, Qishqiya Inkawan Yaku, Tupix (kunkritu rurashqa); imatapis mana maakur kay laaya simintukuna imanawmi tinkun alliq chukruq kaayiyninchawwan hukllaylla chanintsay rurayninchawwan.

Tsay kaayiynintawan kunkritupa chanintsaynintam musyapakushqa, tsaypaq Institutu amirikanu mituduwanmi rurakashqa llapan alli risistinsiyankunata kaayinapaq 21wan $28 \mathrm{Mpa}$ nishqanwan. Tsaypitanam Intiwan Qishqiya Inkawan Tupix nishqanta yakuta yaparkur ruraykunata rurakashqa. Tsaypitanam Brikayta maakinachaw uniyaksiyal kargachaw rurapaayarqan chukruyninta kaayinanpaq, tsaynam llapan chanintsayninta hurqayarqan ayka matiryalkuna tsay kunkritunchaw yaykunqanta. Qipa ushayninchawnam kuntratasiyunta ushayninta rurayarqan, tsay kanaq Qishqiya simintu kunkrituchaw, maa tsaywan alli yarqunaq, tsayraykurnin 21wan 28 Mpa nishqanpaq disiñu rurayninchaw awqaqashqa, hinaman hukllayllaman chaninpis uchukyarqan.

Pushaq shimikuna: kunkritu; yapayninkuna; simintu; chukru tsayraynin; kaayiynin; brikitakunapa chaninkuna.

\section{INTRODUCCIÓN}

En Huaraz, en la construcción de diversas obras se emplea concretos fabricados con agregados de la cantera Tacllán y en forma indiscriminada cementos de las marcas: Sol, Quisqueya e Inka y agua, y topex (concreto listo); sin tener en cuenta que la marca de cemento utilizado repercute en la resistencia a la compresión y en los costos unitarios de fabricación.

En la fabricación de concreto se emplea materiales en las mismas proporciones de peso y relaciones agua/cemento, resultantes del diseño por el método del Instituto Americano del Concreto (ACI) para una determinada resistencia a la compresión, a excepción del topex que es un concreto preparado para una resistencia determinada, teniendo solo que agregarse agua de acuerdo a la especificación del fabricante.

Los profesionales y técnicos dedicados a la industria de la construcción o ejecución de obras donde se emplea concreto no disponen de la información, que diga con cual de los cementos que se fabrica concretos se alcanza mayor resistencia a la compresión y a qué costos unitarios.

Se ha estudiado concretos para resistencias a la compresión de 21 y 28 Mpa y sus costos unitarios de fabricación, teniendo en cuenta el diseño de mezclas de agregados finos y gruesos (Pasquel, 1996) y la resistencia a compresión requerida según las Normas Técnicas Peruanas (NTP). 
La resistencia se ha determinado mediante ensayos de briquetas de concretos (ASTM C-172) usando la máquina de compresión uniaxial a través de la cual se aplica carga hasta que colapsan, con lo cual se calcula la resistencia a la compresión (ASTM C-39).

Los costos unitarios se han determinado teniendo en cuenta los materiales requeridos por $\mathrm{yd}^{3}$ de concreto y precios del mercado.

Determinada la influencia de la marca de cemento en la resistencia a la compresión del concreto y su costo de fabricación, se elige la marca de cemento con la que se obtiene mayor resistencia y los menores costos unitarios.

El estudio tiene relevancia en Huaraz y zonas cercanas, pues involucra a todos los agentes dedicados a la construcción de obras en las que se emplea concreto.

Los resultados obtenidos contribuyen a mejorar los aspectos técnicos y económicos en la ejecución de obras en las que se emplea concreto, repercutiendo en el desarrollo local, regional y nacional.

\section{MATERIALES Y MÉTODOS}

El estudio se inicia con la obtención de agregados de la cantera Tacllán, determinándose en laboratorio las características físicas tanto del agregado grueso con el tamaño máximo de $3 / 4 "$, así como del agregado fino, con lo cual se realiza el diseño de mezclas de concreto empleando relación agua cemento para un asentamiento entre 3" a 4"; para las resistencias a la compresión más usadas en la construcción de estructuras de concreto: $\mathrm{F}^{\prime} \mathrm{c}=21 \mathrm{Mpa}$ y $\mathrm{F}^{\prime} \mathrm{c}=28 \mathrm{Mpa}$.

Obtenidos los diseños de mezclas, se fabricaron y elaboran briquetas de concreto empleando cementos: Sol, Quisqueya e Inka y el topex-concreto listo, con medidas y procedimientos estandarizados de acuerdo a las normas, con altura $\mathrm{H}=12$ " y diámetro $\mathrm{D}=6 "$, con una relación de esbeltez de $\mathrm{H} / \mathrm{D}=2$. Se realizó el curado de las briquetas por un periodo de 7 días después de ser desencofradas a las 24 horas.

Las briquetas de concreto se ensayaron en la máquina de compresión uniaxial a los 14, 21 y 28 días, midiéndose la carga axial que soporta cada una de ellas.

Determinada la carga axial que soportan las briquetas de concreto se calcularon las resistencias a la compresión mediante la expresión:

$$
F^{\prime} c=\frac{P}{A}
$$

Donde:

$F^{\prime} c=$ Resistencia a la compresión del concreto

$P \quad=$ Carga que soporta la briqueta de concreto

$A=$ Área de la sección transversal de la briqueta de concreto

La población la constituyen todas las briquetas de concreto fabricadas, según se detalla: Por cada resistencia a la compresión de diseño: F'c $=21 \mathrm{Mpa} \mathrm{y} \mathrm{F'c}=28 \mathrm{Mpa}$ (factor 2); por cada tipo de cemento: Sol, Quisqueya e Inka y el topex (factor 4); por cada periodo de ensayo: 14, 21 y 28 días (factor 3) y número de briquetas por ensayo (factor 3). Hacen un total de: (2) $\mathrm{x}(4) \times(3) \times(3)=72$ briquetas. 
La unidad de análisis y muestra está constituida por la misma población.

Los costos unitarios se determinaron en base a la cantidad de materiales que se requiere por $\mathrm{Yd}^{3}$ de concreto, obtenidos del diseño de mezclas y los precios promedio del mercado.

En cuanto a los insumos para la investigación se utilizó los siguientes materiales:

Cemento Sol. Es un cemento Portland Normal Tipo I, se emplea en la fabricación de concretos que no requieren de propiedades especiales, cumple los requisitos de las NTP 334.039 y Norma Técnica Americana ASTM C 150.

Cemento Quisqueya. Es un cemento Portland, se emplea en la fabricación de concretos que no requieren de propiedades especiales, cumple los requisitos de las NTP 334090 y Norma Técnica Americana ASTM C-595.

Cemento Inka. Es un cemento Portland Normal Tipo I, se emplea en la fabricación de concretos que no requieren de propiedades especiales, cumple con los requisitos de las NTP 334.039 y Norma ASTM C 150.

Topex (concreto listo). Es una mezcla seca de arena gruesa, piedra y cemento (concreto premezclado), listo para agregarle la cantidad especificada por el fabricante.

Agregados. Materiales inertes divididos en finos y gruesos, sus características influyen en la resistencia del concreto (Pasquel, 1996).

Agua Potable. Utilizada para la hidratación del cemento y el desarrollo de resistencia del concreto, no debe contener sustancias dañinas (ITINTEC-NTP 339.088).

Concreto. Producto artificial resultante de la mezcla de cemento, agregados (fino y grueso) y agua, cuyas proporciones están en función de la resistencia a la compresión requerida (Rivva, 2000).

\section{RESULTADOS}

La cantera Tacllán, está ubicada al lado sur de la ciudad de Huaraz, distrito de Huaraz, provincia de Huaraz, departamento de Áncash. La explotación de la cantera se realiza a tajo abierto, según se observa en las vistas panorámicas en las figuras 1 y 2.

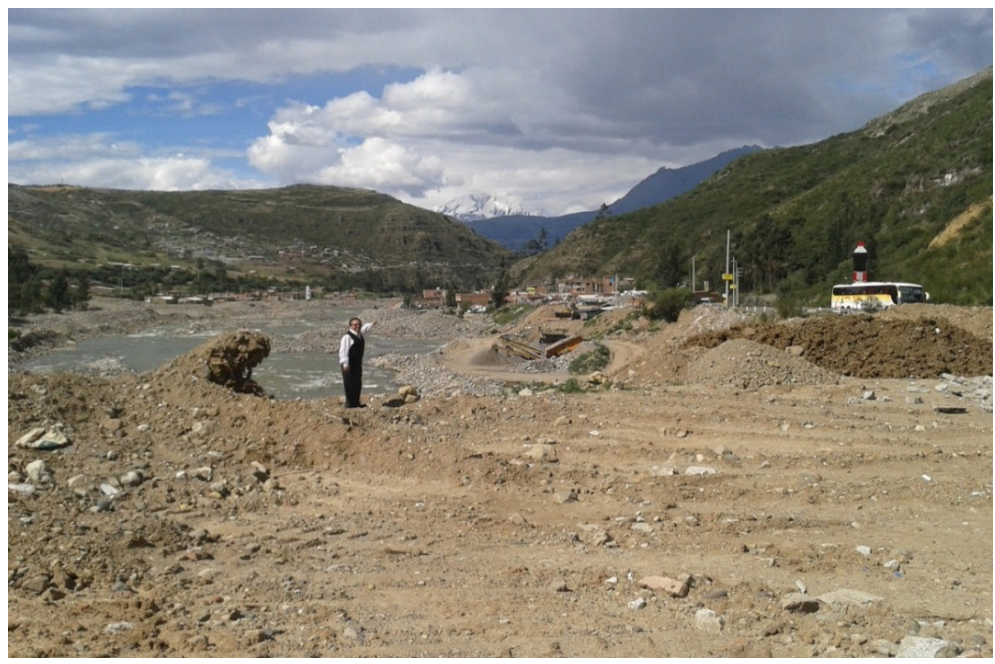

Figura 1. Vista panorámica de la cantera Tacllán en el cauce del río Santa 


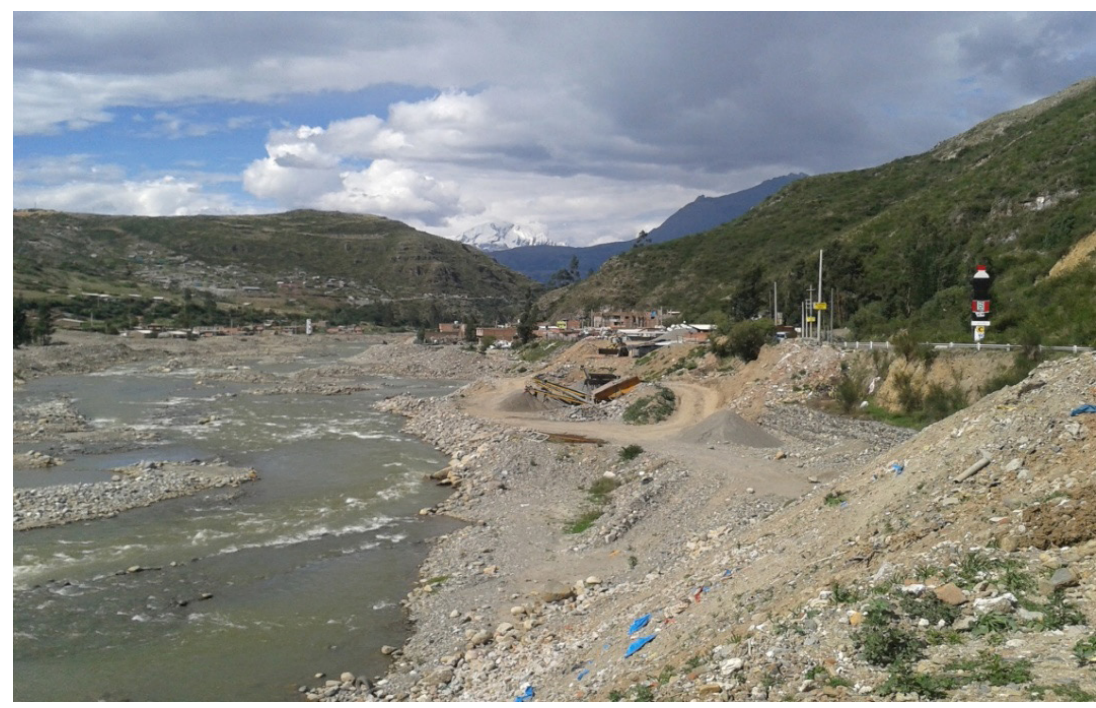

Figura 2. Vista panorámica de la cantera Tacllán en el cauce del río Santa

Las características físicas de los agregados son observados en la tabla 1.

Tabla 1. Características físicas de los agregados

\begin{tabular}{|c|c|c|c|c|c|c|}
\hline $\begin{array}{c}\text { Tipo } \\
\text { de Agregado }\end{array}$ & $\begin{array}{c}\text { Contenido } \\
\text { Humedad } \\
(\%)\end{array}$ & $\begin{array}{c}\text { Absorción } \\
(\%)\end{array}$ & $\begin{array}{c}\text { Peso } \\
\text { Específico }\end{array}$ & $\begin{array}{l}\text { Módulo } \\
\text { de } \\
\text { Fineza }\end{array}$ & \multicolumn{2}{|c|}{$\begin{array}{c}\text { Pesos Unitarios } \\
\text { (Lb/yd3) } \\
\text { Suelto Compactado }\end{array}$} \\
\hline Arena de río & 3,08 & 2,43 & 2,59 & 3,00 & 2836 & 3079 \\
\hline Canto Rodado & 0,58 & 0,76 & 2,66 & 7,56 & 2252 & 2600 \\
\hline
\end{tabular}

Los diseños de mezclas de concreto son observados en la tabla 2.

Tabla 2. Diseños de mezclas para las resistencias: $\mathrm{F}^{\prime} \mathrm{c}=21 \mathrm{Mpa}$ y $28 \mathrm{Mpa}$

\begin{tabular}{|c|c|c|c|c|c|c|}
\hline \multirow{2}{*}{ 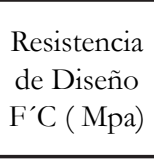 } & \multirow{2}{*}{$\begin{array}{c}\text { Relación } \\
\text { A/C }\end{array}$} & \multirow{2}{*}{$\begin{array}{l}\text { Tipo de } \\
\text { Agregado } \\
\text { Fino }\end{array}$} & \multirow{2}{*}{$\begin{array}{c}\text { Tipo de } \\
\text { Agregado } \\
\text { Grueso } \\
\text { Tam. Máx. } 3 \text { /4» }\end{array}$} & \multirow{2}{*}{$\begin{array}{c}\text { Materiales por } \\
\mathrm{yd} 3 \text { de concreto } \\
\text { (Lb) }\end{array}$} & \multicolumn{2}{|c|}{ PROPORCIONES } \\
\hline & & & & & Peso & Volumen \\
\hline & & & & Cemento: 628 & 1,00 & 1,00 \\
\hline \multirow[t]{4}{*}{21} & 0,55 & Arena de río & Canto rodado & Arena: 1382 & 2,20 & 1,20 \\
\hline & & & & Piedra: 1570 & 2,50 & 1,20 \\
\hline & & & & Agua: 339 & 0,55 & $22,90 \mathrm{~L} / \mathrm{bol}$ \\
\hline & & & & Cemento: 759 & 1,00 & 1,00 \\
\hline \multirow[t]{3}{*}{28} & 0,46 & Arena de río & Canto rodado & Arena: 1467 & 1,90 & 1,70 \\
\hline & & & & Piedra: 1570 & 2,10 & 2,30 \\
\hline & & & & Agua: 339 & 0,46 & $19,00 \mathrm{~L} / \mathrm{bol}$ \\
\hline
\end{tabular}

Las briquetas de concreto cilíndricas, se han fabricado con materiales resultantes de los diseños de mezclas, empleando proporciones en peso; el curado se realizó utilizando agua, según se observa en las figuras 3, 4 y 5. 


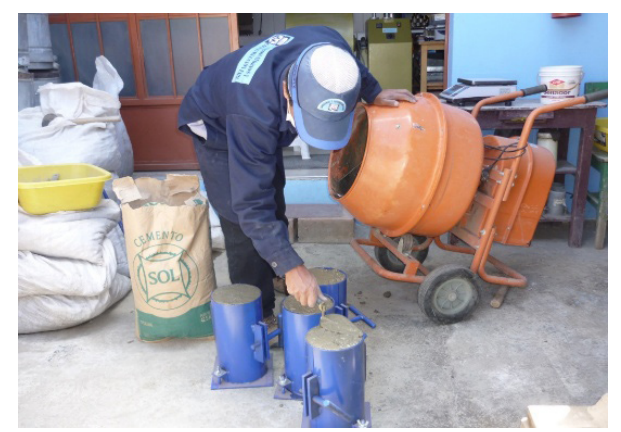

Figura 3. Fabricación de briquetas de concreto con cemento Sol

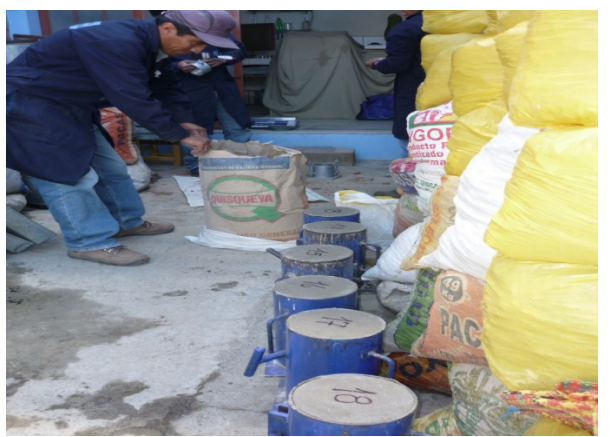

Figura 4. Fabricación de briquetas de concreto con cemento Quisqueya

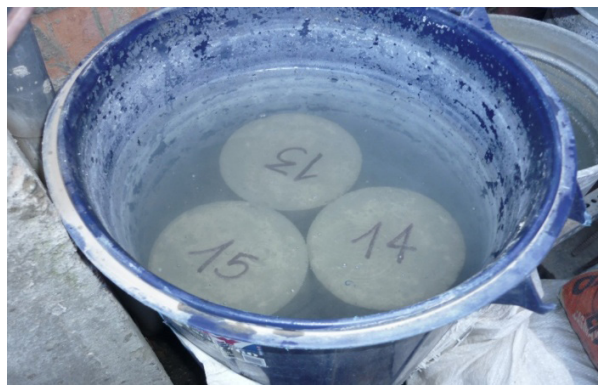

Figura 5. Curado de briquetas de concreto con agua

El ensayo de briquetas de concreto se realizó en la máquina de compresión uniaxial, determinándose la carga de rotura, lo cual se observa en la figura 6.

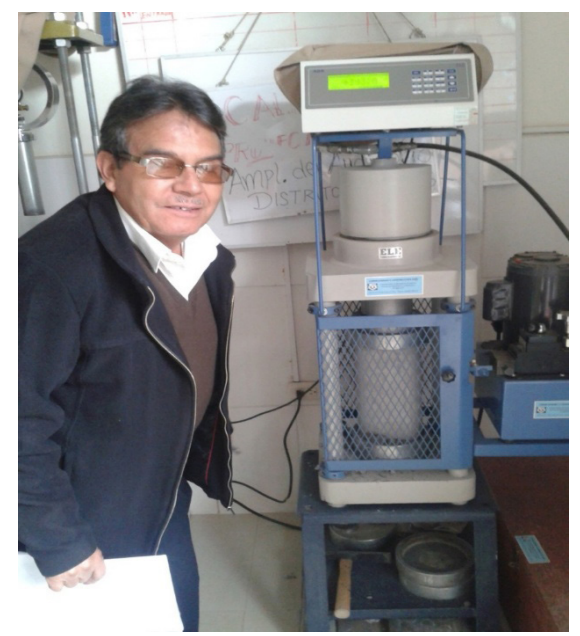

Figura 6. Ensayo de briquetas de concreto a la compresión 
Las resistencias de concretos fabricados con cementos y topex, curados 07 días con agua y ensayados a los 28 días de edad, se observa en la tabla 3.

Tabla 3. Resistencias de concretos ensayados a los 28 días de edad

\begin{tabular}{ccccc}
\hline \multirow{2}{*}{ Cemento } & $\begin{array}{c}\text { Resistencia de } \\
\text { Diseño }(\mathrm{Mpa})\end{array}$ & $\begin{array}{c}\text { Relación } \\
(\mathrm{A} / \mathrm{C})\end{array}$ & $\begin{array}{c}\text { Tipo } \\
\text { Curado }\end{array}$ & $\begin{array}{c}\text { Resistencia a los } \\
28 \\
\text { días }(\mathrm{Mpa})\end{array}$ \\
\hline \multirow{2}{*}{ Sol } & 21 & 0,55 & Agua & 32,843 \\
& 28 & 0,46 & Agua & 38,870 \\
Quisqueya & 21 & 0,55 & Agua & 41,478 \\
& 28 & 0,46 & Agua & 42,417 \\
\multirow{2}{*}{ Inka } & 21 & 0,55 & Agua & 34,187 \\
& 28 & 0,46 & Agua & 35,757 \\
& 21 & 0,55 & Agua & 21,103 \\
Topex & 28 & 0,46 & Agua & ------- \\
\hline
\end{tabular}

Los costos unitarios de materiales de los concretos fabricados con cementos y topex, se observan en la tabla 4.

Tabla 4. Costos unitarios de materiales para fabricación de concretos

\begin{tabular}{ccc}
\hline $\begin{array}{c}\text { Resistencia de Diseño } \\
(\mathrm{Mpa})\end{array}$ & $\begin{array}{c}\text { Marca de Cementos } \\
\text { utilizados y Topex }\end{array}$ & $\begin{array}{c}\text { Costos unitarios de } \\
\text { Materiales }(\mathrm{s} / \mathrm{yd} 3)\end{array}$ \\
\hline 21 & Sol & 198.55 \\
& Quisqueya & 190.19 \\
& Inka & 191.58 \\
& Topex & 354.73 \\
28 & Sol & 220.43 \\
& Quisqueya & 210.58 \\
& Inka & 212.97 \\
& Topex & ------- \\
\hline
\end{tabular}

\section{DISCUSIÓN}

Para un concreto cuya resistencia de diseño es de $21 \mathrm{Mpa}$, las resistencias a la compresión que se logran con el empleo de los diferentes cementos son: Quisqueya 41.48 Mpa; Sol 32.84 Mpa; Inka 34.19 Mpa y topex 21.10 Mpa, a los 28 días de edad.

Para un concreto cuya resistencia de diseño es de $28 \mathrm{Mpa}$, las resistencias a la compresión que se logran con el empleo de los diferentes cementos son: Quisqueya 42.42 Mpa; Sol 38.87 Mpa; Inka 35.76 Mpa, a los 28 días de edad.

Topex-Concreto listo, solo existe en el mercado para resistencia de diseño de $21 \mathrm{Mpa}$, motivo por el cual no se muestra valores para resistencia $28 \mathrm{Mpa}$.

Para un concreto cuya resistencia de diseño es de $21 \mathrm{Mpa}$, los costos unitarios que se determinaron con el empleo de los diferentes cementos son: Quisqueya 190.19; Sol 198.55; Inka 191.58 y topex 354.73 nuevos soles, respectivamente. 
Para un concreto cuya resistencia de diseño es de $28 \mathrm{Mpa}$, los costos unitarios que se determinaron con el empleo de los diferentes cementos son: Quisqueya 210.58; Sol 220.43; Inka 212.97 nuevos soles, respectivamente.

Para concretos cuyas resistencias de diseño son 21 y $28 \mathrm{Mpa}$, empleando cemento Quisqueya se logra mayores resistencias.

Para concretos cuyas resistencias de diseño son 21 y $28 \mathrm{Mpa}$, empleando cemento Quisqueya se logra menores costos unitarios.

\section{CONCLUSIONES}

Los agregados fino y grueso tienen: humedad, absorción, pesos unitarios sueltos y compactados diferentes, y pesos específicos similares.

En el concreto para resistencias de diseño $21 \mathrm{Mpa}$ y $28 \mathrm{Mpa}$, las mayores resistencias a la compresión se logran empleando en su fabricación cemento Quisqueya.

En el concreto para resistencias de diseño $21 \mathrm{Mpa} \mathrm{y} 28 \mathrm{Mpa}$, los menores costos unitarios de materiales para su fabricación se logran empleando cemento Quisqueya.

\section{AGRADECIMIENTOS}

A la Universidad Nacional «Santiago Antúnez de Mayolo», en particular a la Facultad de Ingeniería Civil, por apoyarnos en nuestro perfeccionamiento permanente y así contribuir al desarrollo del país; en nuestro corazón hay sentimientos de gratitud a quienes laboran en ella, es aquí donde existen personas nobles, con valores morales, éticos y humanistas; dispuestas a brindar apoyo en forma desinteresada.

\section{REFERENCIAS BIBLIOGRÁFICAS}

ITINTEC-NTP 339.088. Limites permisibles de sustancias perjudiciales para el agua de mezcla y curado del concreto.

Ministerio de Vivienda, Construcción y Saneamiento. 2009. Reglamento Nacional de Edificaciones. Norma Técnica Peruana E 070. Estructuras de Concreto.

Norma ASTM C-150: Requisitos de los Cementos Pórtland en el Perú.

Norma ASTM C-172: Requisitos para la elaboración de briquetas de concreto.

Norma ASTM C-39. Requisitos para determinar la resistencia a la compresión del concreto.

Pasquel, Enrique. 1998. Tópicos de Tecnología del Concreto. 2da. ed. Lima: Colegio de Ingenieros del Perú - Consejo Nacional.

Rivva, Enrique. 2000. Naturaleza y Materiales del Concreto, Perú. Capítulo Peruano del Instituto Americano del Concreto (ACI).

Fecha de recepción: 10 de febrero de 2015

Fecha de aceptación: 22 de mayo de 2015

\section{Correspondencia}

Víctor Villegas Zamora

viza20@hotmail.com 